\title{
¿SON LOS ENUNCIADOS JURÍDICOS PROPOSICIONES NORMATIVAS?*
}

\author{
Pablo E. Navarro \\ CONICET, Argentina
}

RESUMEN. El conocimiento del derecho es básicamente la aptitud para distinguir entre enunciados jurídicos verdaderos y falsos acerca del derecho de una determinada comunidad. Tales enunciados son un tipo de enunciados normativos y se refieren a las características normativas (i. e., obligatoria, permitida) de una cierta acción. En su trabajo clásico acerca de los sistemas normativos, ALCHOURRÓn y BULYGIN ofrecen el análisis más elaborado de la naturaleza de los enunciados jurídicos. Básicamente, ellos señalan que los enunciados jurídicos son proposiciones normativas disfrazadas, i. e., que se refieren de manera oblicua a la existencia de normas. En su opinión, este reduccionismo está vinculado a una concepción positivista del derecho. En este trabajo, sin embargo, se pretende mostrar no sólo diferencias importantes en las distintas caracterizaciones que ALCHOURRÓN y BULYGIN han ofrecido de los enunciados jurídicos y las proposiciones normativas sino también que un positivista jurídico no está compelido a suscribir su análisis clásico.

Palabras clave: enunciados jurídicos, proposiciones normativas, positivismo jurídico.

ABSTRACT. The cognition of the law of a certain community requires distinguishing between true and false legal statements. Such statements are a type of normative statements, and normative statements describe that certain actions are obligatory (forbidden) or permitted. In their classical book on normative systems, ALCHOURRÓN and BULYGIN offer the most elaborated analysis of the nature of legal statements. Basically, they claim that legal statements are disguised normative propositions (that is, propositions that refer to the existence of legal norms) and their reductionism is grounded on a specific conception of law and legal theory: legal positivism. In this paper, I claim not only that ALCHOURRÓN and BULYGIN actually endorsed different views about legal statements and normative propositions but also that a legal positivist is not committed to subscribe the classical analysis offered by them.

Keywords: legal statements, normative propositions, legal positivism.

* Fecha de recepción: 4 de diciembre de 2012. Fecha de aceptación: 21 de diciembre de 2012.

Este texto es una versión revisada (y reducida) de la ponencia presentada al XVII Seminario HispanoItalo-Francés de Teoría del Derecho, celebrado en la Universidad Carlos III de Madrid en 2011. En la ponencia original, se analizaba el problema de la fuerza de los permisos normativos y su conexión con los enunciados jurídicos y las proposiciones normativas. Los comentarios y discusiones en esa reunión, junto a otras críticas de colegas en ocasiones posteriores, me han persuadido de dividir la versión original en dos trabajos diferentes y en este artículo he dejado de lado el problema de las permisiones normativas. Agradezco a J. M. ${ }^{a}$ SAUCA y los editores de Doxa la oportunidad de reproducir aquí este trabajo. También agradezco a E. BuLYGin sus comentarios a una versión previa de este artículo. 


\section{INTRODUCCIÓN}

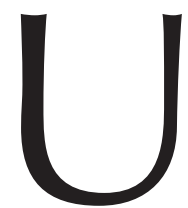

no de los rasgos paradigmáticos del derecho es que impone obligaciones. La existencia del derecho, señala HART al inicio de su obra más célebre, «significa que ciertos tipos de conducta humana no son ya optativos sino obligatorios» ${ }^{1}$. Esta característica de los ordenamientos jurídicos exige una explicación de la naturaleza de las obligaciones que surgen del derecho y es una de las preocupaciones centrales de la teoría del derecho. KELSEN, por ejemplo, subrayaba que, en general, el factor determinante de una obligación, i. e., aquello que convertía a una mera exigencia en un genuino deber, dependía de una propiedad específica: la validez ${ }^{2}$. Este enfoque es aun influyente en la teoría jurídica contemporánea. Por ejemplo, para RAz, las normas válidas son normas, mientras que no hay —salvo en las extravagancias del lenguaje- espacio para admitir normas inválidas ${ }^{3}$. El derecho es normativo porque sus normas imponen genuinas obligaciones, lo que equivale a decir que sus normas son válidas.

La conexión entre normatividad y derecho genera una peculiar concepción de la ciencia jurídica en la que los mismos enunciados de los juristas —y no sólo las normasson entendidos, por ciertos teóricos influyentes, como normativos ${ }^{4}$. Sin embargo, esta manera de reconstruir a la ciencia jurídica no es unánime y sigue siendo un problema abierto en la teoría jurídica determinar claramente la naturaleza de los enunciados jurídicos. Estos enunciados son una clase de enunciados normativos y, siguiendo a VON WRIGHT, puede decirse que los enunciados normativos afirman que una determinada acción es obligatoria, prohibida o permitida (e.g. «Está prohibido conducir a más de 110 kilómetros por hora»). En general, se admite que el fundamento de la verdad de un enunciado normativo es la existencia de una norma y, a su vez, es usual señalar que una proposición que afirma que una cierta norma existe es una proposición normativa ${ }^{5}$. Por consiguiente, si la tarea central de los juristas es dar cuenta de las obligaciones (prohibiciones o permisiones) en un cierto ordenamiento jurídico, podría asumirse que esta tarea es satisfecha mediante la identificación de las proposiciones normativas verdaderas referidas a un particular ordenamiento jurídico.

Sin embargo, el esfuerzo por clarificar la noción de proposición normativa ha generado un persistente - y con cierta frecuencia, infructuoso- debate en teoría del derecho. En particular, dos cuestiones son especialmente relevantes en el análisis de este tema. Por una parte, la distinción entre normas y proposiciones normativas. Por otra parte, la distinción entre proposiciones normativas y enunciados normativos. A menudo, los juristas vislumbran la necesidad de distinguir tajantemente entre normas y proposiciones normativas, pero, a menudo, ese insight ha sido dejado de lado ${ }^{6}$. A su vez, esta falta de distinción entre normas y proposiciones repercute de manera directa

1 H. L. A. HART, El concepto de derecho, Buenos Aires, Abeledo Perrot, 1964, 7.

2 H. Kelsen, Teoría pura del derecho, México, UNAM, 1979, 23 y ss.

3 J. RAz, «Legal Validity», The Authority of Law, Oxford, Oxford University Press, 1979, 146.

${ }^{4}$ Por ejemplo, Kelsen y, en cierto modo, también HART, RAZ y DwORKIN.

5 G. H. von Wright, Norm and Action, London, Routledge \& Kegan Paul, 1963, 106.

6 E. Bulygin, «Normas, proposiciones normativas y enunciados jurídicos», en C. AlChOuRRón y E. Bulygin, Análisis lógico y derecho, Madrid, Centro de Estudios Constitucionales, 1991, 169-193. 
en el modo en que se analiza la distinción entre proposiciones normativas y enunciados normativos.

Es bien conocido que uno de los aportes notables de C. AlChOuRRón y E. BulYGIN (en adelante, A-B) a la lógica deóntica y la filosofía del derecho ha sido la caracterización rigurosa de la diferencia entre normas y proposiciones normativas ${ }^{7}$. En el enfoque de A-B hay dos rasgos estrechamente conectados. Por una parte, los enunciados normativos (i. e. afirmaciones acerca de lo que se debe o puede hacer) son vistos como una manera indirecta de afirmar proposiciones normativas y, por otra parte, una de las características destacable de las proposiciones normativas es la explicación de lo que se «puede» hacer a partir de dos conceptos específicos de permisión, denominados «permisión débil» y «permisión fuerte», respectivamente referidos a la ausencia de una norma que prohíbe una cierta acción y la existencia de una norma que autoriza una cierta acción. En gran medida, ambos rasgos contribuyen de manera decisiva a justificar una de las tesis centrales de A-B: la necesidad de elaborar lógicas diferentes para el nivel prescriptivo y descriptivo de los discursos normativos.

Aunque es difícil negar la importancia de los trabajos de A-B, su propuesta ha sido objeto de persistentes críticas $^{8}$. Por ejemplo, en un breve trabajo publicado hace más de una década, A. SoETEMAN elabora una caracterización de las proposiciones normativas diferentes de la que proponen A-B, y subraya la importancia de distinguir entre proposiciones normativas y enunciados normativos - a pesar de que no emplea esa terminología-. SOETEMAN señala que en la distinción de A-B entre normas y proposiciones normativas «hay una cierta ambigüedad... que provoca que ella sea menos evidente de lo que parece» (326). La ambigüedad surge de la siguiente afirmación de $A-B^{9}$ :

Las proposiciones normativas son enunciados descriptivos que se usan para informar acerca de las normas o acerca de las obligaciones, prohibiciones o permisiones establecidas por las normas.

Como ejemplo de la necesidad de distinguir entre la descripción de las normas que forman parte del sistema y la descripción del status normativo de una conducta, SOETEMAN introduce el problema de la fuerza de los permisos. Aunque dos conductas A y B estén débilmente permitidas (i. e., no están prohibidas) esa información todavía no dice nada acerca de la «intensidad» con que esa conducta es permitida por el derecho. Por ello, las proposiciones normativas acerca de las normas que pertenecen a un sistema no garantizan que conozcamos en qué medida (con qué fuerza) podemos hacer determinadas cosas. Para SoETEMAN, el conocimiento del derecho se asocia bási-

7 Sin lugar a dudas, la referencia más importante es C. AlChOuRRón y E. BulYgin, Normative Systems, New York-Wein, Springer, 1971. En adelante, las referencias a ese libro remiten a la versión en castellano: Introducción a la metodología de las ciencias jurídicas y sociales, Buenos Aires, Astrea, 1974. En adelante, IMCJ. La importancia de ese libro no debe ocultar la relevancia de otros trabajos de ALCHOURRÓn y BULYGIN. Así, son especialmente importantes las siguientes obras: C. ALCHOURRÓN y E. BuLYGin, Sobre la existencia de normas jurídicas, Valencia, Venezuela, Universidad de Carabobo, 1979, y C. AlChourrón y E. BulYgin, Análisis lógico $y$ derecho, op. cit.

${ }^{8}$ J. C. BAYÓN, «Sobre el principio de prohibición y las condiciones de verdad de las proposiciones normativas», en E. BulYgin et al., Problemas lógicos en la teoría y práctica del derecho, Madrid, Fundación Coloquio Jurídico Europeo, 2009, 46.

9 C. AlChOurRón y E. Bulygin, IMCJ, op. cit., 173. 
camente a la identificación de calificaciones normativas de la conducta, y ello no puede reducirse a la descripción de las normas que forman parte de un sistema normativo ${ }^{10}$. En otras palabras, los enunciados normativos no pueden ser reducidos a proposiciones normativas.

A pesar de que A-B han insistido de manera constante en la importancia de distinguir entre normas y proposiciones normativas (y en la necesidad de elaborar lógicas diferentes para cada ámbito de estos discursos), sus concepciones acerca de las relaciones entre proposiciones normativas y enunciados normativos han variado significativamente. En particular, señalaré que A-B han defendido tres versiones diferentes de la relación entre proposiciones normativas y enunciados normativos:

- La identidad de significado entre proposiciones y enunciados normativos.

- La equivalencia material entre proposiciones y enunciados normativos.

- Las proposiciones normativas como implicadas por los enunciados normativos.

Cualquiera de estas tres versiones tiene méritos y defectos. En gran medida, esas ventajas y desventajas muestran nuestros compromisos acerca de la naturaleza del conocimiento jurídico. Por consiguiente, la moraleja de este ensayo sería la siguiente: el énfasis en la diferencia entre normas y proposiciones normativas ha impedido apreciar debidamente el papel que cumplen los enunciados normativos. Por tanto, si hay buenas razones para distinguir entre proposiciones normativas y enunciados normativos, entonces también hay que revisar nuestras ideas acerca de qué conocemos cuando conocemos el derecho.

\section{EL SIGNIFICADO DE LOS ENUNCIADOS NORMATIVOS}

En general, los filósofos del derecho creen que la verdad de los enunciados normativos está conectada con la verdad de otras proposiciones. Por ejemplo, en las primeras páginas de Law's Empire, DwORKIN señala que los enunciados normativos son verdaderos o falsos en virtud de la verdad o falsedad de otras proposiciones, más familiares, que suministran el fundamento de los enunciados normativos ${ }^{11}$. ¿Qué relación existe entre los enunciados normativos y sus fundamentos? Al respecto, es ilustrativa la siguiente afirmación de $\mathrm{A}-\mathrm{B}^{12}$ :

Las proposiciones normativas proporcionan información sobre el status deóntico de ciertas acciones o estados de cosas: enuncian que una acción es obligatoria, prohibida o permitida, y son verdaderas si, y sólo si, la acción en cuestión tiene la propiedad de ser obligatoria, prohibida o permitida. Pero, ¿cuándo una acción tiene la propiedad de ser obligatoria? Esta pregunta admite distintas respuestas. Algunos filósofos creen que la obligatoriedad es una propiedad intrínseca (probablemente no natural) de la acción y su presencia puede ser

10 A. Soeteman, «On Legal Gaps», en E. Garzón ValdÉs et al. (eds.), Normative Systems in Legal and Moral Theory. Festschrift for Carlos Alchourrón and Eugenio Bulygin, Berlin, Duncker, 1997, 323-332. En gran parte, este artículo está reproducido en la entrada «Legal Gaps» del diccionario online de la IVR. Vid. A. SoETEMAN, «Legal Gaps», IVR-Encyclopedie, 2009, http://ivr-enc.info/index.php?title=Legal_gaps.

11 R. Dworkin, Law's Empire, Cambridge, Mass., Harvard University Press, 1986, 4.

12 C. Alchourrón y E. Bulygin, «Von Wright y la filosofía del derecho», en C. Alchourrón y E. BulyGIN, Análisis lógico y derecho, op. cit., 88. 
detectada mediante el examen detenido de la acción. Otros filósofos creen que una acción es obligatoria en virtud de mandatos divinos o ciertos principios eternos del derecho natural. Pero nosotros estamos interesados en el derecho positivo y en el enfoque positivista que es compartido por la mayoría de los juristas (aunque no por los filósofos del derecho). La característica distintiva de este enfoque es que la acción $p$ es obligatoria si y sólo si hay una norma (en el derecho positivo del país en cuestión) que ordena $p$ (o prohíbe no $p$ ), y no porque tal acción sea intrínsecamente buena o calificada así por principios morales o de derecho natural. Esto equivale a decir que la proposición de que $p$ es obligatoria significa lo mismo que la proposición de que hay (existe) una norma jurídica que ordena hacer $p$.

En este párrafo, se expresa claramente lo que podría denominarse como la posición clásica de A-B: los enunciados normativos tienen el mismo significado que las proposiciones normativas. En este enfoque clásico, A-B definen a los enunciados normativos en función de la pertenencia de normas a un cierto sistema normativo. Para A-B la expresión descriptiva «Op $\mathbf{O}$ » es definida en términos de la proposición «Op $\varepsilon$ Cn $\alpha »$. Así, ellos señalan ${ }^{13}$ :

Decir que $p$ está prohibido en el sistema $\alpha$ es afirmar que la norma que prohíbe $p$ forma parte (es consecuencia, se infiere) del sistema $\alpha$. Por tanto, el enunciado metasistemático «p está prohibido en $\alpha »$ es verdadero si y sólo si, entre las consecuencias de $\alpha$ se halla una norma que prohíbe $p$.

En la medida en que una definición introduce una equivalencia de significados, los enunciados normativos quedan «reducidos» a proposiciones y ellas «son normativas sólo en el sentido de que lo que ellas describen son normas o, más bien, la existencia de normas» ${ }^{14}$.

Según se ha señalado, A-B reivindican un enfoque positivista del derecho como explicación de «reducir» los enunciados normativos a proposiciones normativas. Sin embargo, en el heterogéneo campo del positivismo jurídico es posible encontrar otras respuestas, que rechazan esa identidad de significado. Veamos este punto con cierto detalle.

En gran medida, los esfuerzos de las diferentes doctrinas positivistas están guiadas por el propósito de comprender adecuadamente dos cosas: a) si los términos «obligación», «deber», «derechos», etc., tienen diferente significado en la moral y el derecho, y b) si el significado de los términos «obligación, «deber», «derechos», etc., puede ser explicado mediante la referencia a cuestiones fácticas. En este complejo panorama, el denominado «positivismo simple» ha señalado la necesidad de explicar el significado de los enunciados normativos en referencia a proposiciones empíricas, como por ejemplo, enunciados acerca de la probabilidad de aplicación de un determinado castigo $^{15}$.

Una reacción contra esa propuesta ha conducido a un enfoque anti-reduccionista. H. L. A. HART puede ser considerado como uno de los ejemplos paradigmáticos de esta propuesta teórica ${ }^{16}$. En su célebre libro The Concept of Law, HART distingue en-

13 C. AlChOuRRón y E. BuLYGIn, IMCJ, op. cit., 174.

${ }^{14}$ E. Bulygin, El positivismo jurídico, México, Fontamara, 2006, 77.

15 La denominación «Positivismo simple» es la que usa R. SHINER para reconstruir la teoría de AusTiN y Bentham. Al respecto, vid. R. SHINER, Norm and Nature, Oxford, Oxford University Press, 1992.

16 Otro conocido exponente es H. KeLSEN. Sin embargo, no es posible analizar su doctrina en los límites de este trabajo. Para una excelente presentación general de las ideas de KELSEN y una cuidadosa discusión del 
tre enunciados internos y externos, pero su reconocido énfasis en el papel de los que adoptan el punto de vista interno ha llevado, con frecuencia, a asumir que la descripción del derecho sólo puede darse en términos de enunciados internos. Al respecto, BULYGIN señala ${ }^{17}$ :

Creo que se trata de un error, pero no estoy seguro de que HART no tenga parte de la culpa. Es un error porque lo que HART ataca no es el uso exclusivo de enunciados externos en la descripción del derecho, sino el uso exclusivo de enunciados externos... que sólo registran las regularidades de conducta. Pero HART no dice qué pasa con aquel que describe el derecho mediante... enunciados que registran el hecho de que los miembros de la sociedad consideran a las reglas como tales.

BULYGIN considera que los enunciados internos son «prescripciones disfrazadas» y que no se pueden aceptar en una teoría descriptiva del derecho como la defendida por el positivismo jurídico ${ }^{18}$. A su vez, admite que ciertos enunciados externos (i.e., aquellos que no sólo registran regularidades de conducta sino que también dan cuenta de las reglas de un grupo social) pueden dar cuenta de las obligaciones y derechos de los miembros de una cierta comunidad.

Esta solución (i. e., el énfasis en el papel de los enunciados externos en la descripción del derecho) es la que parece adoptar HART en sus últimos trabajos. Por ejemplo, en la introducción a su colección de ensayos sobre derecho y filosofía, escribe que en The Concept of Law sostuvo erróneamente que el vocabulario normativo (e. g. expresiones tales como «deber», «tener», «obligación») fuesen adecuadamente usados sólo en enunciados internos. Allí, HART añade ${ }^{19}$ :

Esto es un error porque, por supuesto, tales términos son claramente usados adecuadamente en otras formas de enunciados, y particularmente en los enunciados de los juristas de las obligaciones o deberes, que describen el contenido de los sistemas jurídicos (ya sea el sistema propio o uno ajeno), cuyas reglas ellos de ningún modo aceptan o apoyan como estándares de conducta. Al hacer esto, los juristas informan en una forma normativa el contenido de una norma desde el punto de vista de aquellos que aceptan las normas sin que ellos mismos compartan ese punto de vista.

Sin embargo, como veremos a continuación, la solución de HART es diferente a la posición clásica de A-B. En un artículo publicado en su colección de ensayos sobre BENTAHM, HART distingue entre enunciados del derecho y enunciados acerca del derecho ${ }^{20}$. Aunque sería tentador imaginar que esa distinción es la misma que la que HART había elaborado entre enunciados internos y externos, creo que más pertinente analizar esa distinción como la explicación del papel que diferentes tipos de enunciados externos juegan en la descripción del derecho. Según HART, los enunciados del derecho $^{21}$ :

problema de los enunciados jurídicos en la Teoría pura del derecho, vid. B. CELANO, La teoría del diritto di Hans Kelsen, Milano, Il Mulino, 1999.

17 E. Bulygin, «Alf Ross y el realismo escandinavo», Anuario de Filosofía Jurídica y Social, I, 1981, 88.

18 E. BulYGin, «Normas, proposiciones normativas y enunciados normativos», en C. AlCHOURRÓN y E. Bulygin, Análisis lógico y derecho, op. cit., 185.

${ }^{19}$ H. L. A. HART, «Introduction», en Essays in Jurisprudence and Philosophy, Oxford, Oxford University Press, 1983, 14.

${ }^{20}$ H. L. A. HART, «Legal Duty and Obligation», Essays on Bentham, Oxford, Oxford University Press, 1982.

${ }^{21}$ Ibid., 144. 
[...] son la manera más común de establecer el contenido del derecho, en relación a cualquier tema, por parte de los ciudadanos, abogados, jueces u otros funcionarios, y también por los juristas y los profesores de derecho en relación a sistemas jurídicos propios o ajenos.

Este párrafo sugiere que los enunciados del derecho no comprometen con un punto de vista interno acerca de la corrección de las pautas de conducta exigidas. Al menos parece extraño suponer que un profesor de derecho que informa sobre el contenido de un sistema jurídico extranjero lo hace mediante enunciados internos que expresan su aceptación de las pautas en cuestión.

En general, HART subraya el fracaso de los intentos de analizar el significado de los enunciados del derecho mediante la explicación del significado de enunciados morales. A su vez, HART también rechaza la equiparación entre enunciados del derecho y enunciados acerca del derecho. En este sentido sostiene que los enunciados del derecho ${ }^{22}$ :

[...] no son enunciados históricos o fácticos que describan acciones presentes, pasadas o futuras, actitudes o creencias ya sea de los individuos o de los funcionarios del sistema jurídico, sino enunciados de lo que los individuos jurídicamente deben o no deben bacer (cursivas añadidas).

¿Qué relación existe, entonces, entre los enunciados del derecho y acerca del derecho? HART no niega que los juristas usan enunciados fácticos o históricos acerca del derecho. Así, en lugar de decir que los mayores de edad están obligados a empadronarse, con frecuencia se afirma que el parlamento aprobó una determinada norma que exige esa conducta. Sin embargo, según HART ${ }^{23}$,

[...] ese sería un enunciado histórico acerca del derecho y no un enunciado de derecho, pero aunque ambas formas de enunciados sean verdaderas y estén íntimamente conectadas ellas no tienen el mismo significado. El enunciado histórico acerca del derecho, que el Parlamento promulgó una ley particular, establecen hechos que son parte de las condiciones de verdad del enunciado normativo del derecho, pero esa conexión entre los dos enunciados no debe ocultar sus diferentes significados.

De esta manera, a diferencia de la posición clásica de A-B, para HART los enunciados que describen el status deóntico de una acción no tienen el mismo significado que las proposiciones acerca del derecho, ni pueden ser reducidos a afirmaciones acerca de lo que ha prescrito una determinada autoridad. La misma idea es subrayada por RAZ cuando señala que la relación entre el enunciado acerca de la existencia de una fuente normativa y el correspondiente enunciado jurídico no es de identidad de significado sino más bien la que existe entre fundamento y consecuencia ${ }^{24}$.

En este sentido, RAZ afirma ${ }^{25}$ :

La existencia de una fuente es el fundamento de la verdad de un enunciado acerca de lo que se debe jurídicamente hacer, de la misma manera que la existencia de una instancia apropiada es el fundamento de la verdad de un enunciado existencial.

22 Ibid., 144.

23 Ibid., 145.

24 J. RAZ, «Legal Reasons, Sources and Gaps», op. cit., 65. Sin embargo, el anti-reduccionismo de RAZ se distingue del de HART y KELSEN en que RAZ no niega que las expresiones «obligatorio», «deber», «derechos», etc., tengan el mismo significado en contextos morales y jurídicos.

25 J. RAZ, «Legal Reasons, Sources and Gaps», op. cit., 66. 
En conclusión, A-B introducen su posición clásica como una elaboración comprometida con un enfoque positivista del derecho. Sin embargo, otros representantes notables de esta doctrina (e. g., KELSEN, HART y RAZ) subrayan la diferencia en el significado de los enunciados normativos y otras proposiciones parasitarias que contribuyen a su fundamento. En este sentido, la apelación al positivismo jurídico no puede dirimir la polémica acerca de la relación entre enunciados y proposiciones normativas.

\section{NORMAS, ENUNCIADOS Y PROPOSICIONES}

La posición clásica de A-B podría defenderse señalando que la diferencia entre enunciados normativos y proposiciones normativas es más bien retórica y se podría explicar el uso del lenguaje normativo como un modo material de hablar. El lenguaje normativo sería, como señala CARNAP, algo así como una suerte de manera transpuesta de expresarse «en el que para decir algo acerca de un objeto $a$, decimos algo paralelo acerca de un objeto $b$ que está en una relación determinada con el objeto $a{ }^{26}$. Por ello, ante la pregunta «¿Qué diferencia podría haber entre afirmar, por ejemplo, que hay una norma que prohíbe estacionar en un sitio $S$ y afirmar que está prohibido estacionar en S?», BuLYGIN responde que no hay diferencias. Por ello señala ${ }^{27}$ :

¿Cómo podría informar a alguien acerca de las regulaciones existentes más que diciéndole que hay una norma que prohíbe estacionar aquí? Si mi información es verdadera, entonces hay una norma a los efectos de que estacionar es prohibido; y si no hay tal norma, entonces mi enunciado normativo es falso. En contra de lo que puede parecer, ambos enunciados dicen exactamente lo mismo. En otras palabras, aún si el enunciado normativo que dice que estacionar está prohibido parece referirse a la acción de estacionar, y la proposición normativa parece referirse a la existencia de una norma, ambos tienen exactamente las mismas condiciones de verdad y, más aún, ellos tienen el mismo significado.

Hace una década presenté una crítica a la posición clásica de $\mathrm{A}-\mathrm{B}^{28}$, y poco después, en ocasión de reimprimir este artículo en castellano, BuLYGIN concede que es necesario abandonar esta tesis clásica. No tiene mayor sentido reproducir aquí mis observaciones, pero vale la pena señalar que en esta publicación en castellano sobre el papel de la verdad en el discurso normativo, BULYGIN señala que la posición clásica es «probablemente una exageración» que no está dispuesto a mantener ${ }^{29}$. En cambio, BulYGIN adopta una tesis más débil: los enunciados normativos y las proposiciones normativas son materialmente equivalentes, i. e., tienen las mismas condiciones de $\operatorname{verdad}^{30}$.

Esta nueva concepción de las relaciones entre proposiciones normativas y enunciados normativos introduce cambios que no son menores. Si, por definición, un

26 R. CARnaP, The Logical Syntax of Language, London, Routledge \& Kegan, Paul, 1958, 308.

27 E. Bulygin, «True or False Statements in Normative Discourse», en R. EIGIDI (ed.), In Search of a New Humanism, Dordrecht, Kluwer, 1999, 184.

28 P. NAVArRo, «Legal Reasoning and Systematization of Law», en A. Soeteman (ed.), Pluralism and Law, Boston, Kluwer, 2001, 251-277.

${ }_{29}$ E. Bulygin, «El papel de la verdad en el discurso normativo», Doxa, 26, 2003, 81, nota 3.

${ }^{30}$ Ibid., 83. 
enunciado normativo y una proposición normativa tuviesen el mismo significado, entonces la verdad de una de ellas es necesariamente equivalente a la verdad de la otra. Pero, cuando ellas son sólo materialmente equivalentes nada conecta necesariamente al enunciado normativo que describe que una acción es obligatoria (i.e. «OP $\gg)$ y la proposición normativa que afirma que existe una determinada norma en un cierto sistema normativo (i. e., « $\mathbf{O p} \varepsilon$ Cn $\alpha \gg)$. Así, al romperse la conexión de significado entre enunciados y proposiciones, es natural formular el siguiente interrogante: ¿por qué la verdad de un enunciado normativo depende de la verdad de una proposición normativa? ¿En qué medida contribuye la existencia de normas a la verdad de los enunciados normativos?

Para dar una respuesta satisfactoria a estas preguntas conviene tomar una cierta distancia del discurso específicamente jurídico y analizar el problema en términos más generales. En mi opinión, una gran contribución de VON WRIGHT a la comprensión del discurso normativo en general (como algo diferente al discurso jurídico, que es el que parece interesarle a BULYGIN específicamente) ha sido mostrar en qué sentido la verdad de nuestras afirmaciones sobre obligaciones y deberes morales están fundadas en necesidades individuales y sociales. Para VON WRIGHT, nuestros enunciados normativos morales pueden tener una fundamentación teleológica como diferente a una fundamentación legalista, asociada a la existencia de normas. En su opinión, la diferencia entre una concepción legalista y una teleológica es la siguiente ${ }^{31}$ :

La primera considera a la fundamentación de los enunciados normativos en la existencia de normas como el parámetro de acuerdo con el cual los enunciados de principios morales deben fundarse. La segunda... considera a la fundamentación de los enunciados normativos en necesidades y fines de la acción como el parámetro apropiado.

En otras palabras, VON WRIGHT intenta ofrecer una fundamentación de los enunciados normativos que no depende de la existencia de prescripciones y, en este sentido de «norma», la fundamentación de los deberes morales es independiente de la existencia de normas (i.e., normas entendidas como prescripciones).

Ahora bien, ¿es posible analizar el fundamento de un enunciado jurídico-normativo sin referirse a la existencia de algún tipo de normas? Una respuesta compleja es la que ofrece RAz cuando analiza los enunciados jurídicos en términos de «fuente» sociales y razones para la acción. Aunque RAZ atribuye a las normas (laws) el papel de intermediarios útiles, señala que se puede analizar directamente la conexión entre los hechos que determinan las calificaciones normativas y las razones para la acción, y que esta omisión de las normas no altera ningún aspecto esencial de nuestro discurso ${ }^{32}$. Por el contrario, para BULYGIN, la existencia de normas es una condición indispensable para determinar la verdad de los enunciados normativos. Por ello, BULYGIN sostiene: «El hecho de que una acción sea obligatoria no puede provenir sino de una norma. La existencia de una norma es condición necesaria para que una acción pueda ser calificada de obligatoria» ${ }^{33}$.

31 G. H. von Wright, «The Foundation of Norms and Normative Statements», Practical Reason, Oxford, Blackwell, 1983, 79.

32 J. RAZ, «Legal Reasons, Sources, and Gaps», op. cit., 63.

33 E. BuLYGin, «El papel de la verdad en el discurso normativo», op. cit., 83. Es prudente señalar que la existencia de normas tienen que ser caracterizadas de manera independiente al hecho de que califican deón- 
Es interesante destacar que, a pesar de la supuesta equivalencia material entre enunciados y proposiciones normativas, esta respuesta de BULYGIN deja abierta la posibilidad de que la existencia de una norma que regula una cierta acción no sea suficiente para determinar el status deóntico de una cierta acción. Por ejemplo, en casos de conflictos normativos que pueden resolverse mediante la aplicación de criterios jerárquicos, la afirmación verdadera acerca de la existencia de una norma en un cierto sistema que exige la conducta $\mathrm{R}$ no garantiza que los individuos deban jurídicamente ejecutar R. En este sentido, AlCHOURRÓn afirma ${ }^{34}$ :

[...] no debería sorprendernos el hecho de que las obligaciones determinadas por un sistema de normas cambian cuando se altera su orden jerárquico, aun cuando las mismas normas permanezcan sin alteración. Lo obligatorio está determinado por dos elementos: el contenido conceptual de las normas y su orden jerárquico.

En estos párrafos, la existencia de normas es una condición necesaria para la verdad de un enunciado normativo, pero de allí no se sigue que sean también una condición suficiente. Más bien, si admitimos — como hace ALCHOURRÓN- que hay dos elementos que determinan el status deóntico de la conducta y que la existencia de normas sólo da cuenta de uno de ellos, entonces también hay que abandonar la tesis de la equivalencia material entre enunciados y proposiciones normativas ${ }^{35}$.

\section{CONCLUSIONES}

Este trabajo es un ejercicio de análisis y crítica acerca del conocimiento del derecho. En buena medida, es también un trabajo parcial ya que su principal énfasis es mostrar la necesidad de revisar la distinción entre enunciados normativos y proposiciones normativas, pero no se ofrecen indicaciones precisas sobre las condiciones de verdad de los enunciados normativos ni de su relación con el problema clásico de la «normatividad» de los enunciados de la ciencia jurídica.

La reconstrucción de los enunciados normativos es un problema clásico para las teorías positivistas del derecho ya que, en última instancia, se trata de explicar el vocabulario normativo sin reducir el significado de «deber», «obligación», etc., al significado que ellos tienen en enunciados morales. Según A-B, un enfoque positivista tiene que explicar a ese significado en términos de la existencia de normas en un

ticamente a las acciones. De otra manera, afirmar que la existencia de una norma es condición necesaria de la obligatoriedad de una acción será un enunciado analítico porque parte del significado de «acción obligatoria» es «acción regulada por una norma que exige esa conducta». Pero, una parte importante de nuestra preocupación por la relación entre normas y proposiciones normativa radica en comprender algo más que una relación analítica y, por ello, nos esforzamos en explicar en qué sentido, por ejemplo, la vigencia, la pertenencia o la promulgación de una norma son un fundamento para atribuir obligaciones y derechos.

34 C. Alchourrón, «Condicionalidad y representación de normas jurídicas», en C. AlChOuRRón y E. Bulygin, Análisis lógico y derecho, op. cit., 278.

35 Más allá de la plausibilidad de esta nueva versión de la conexión entre enunciados y proposiciones normativas es importante subrayar que la afirmación: «La existencia de una norma es una condición necesaria para que sea verdad que una acción es obligatoria» es notablemente más débil que sostener: i) la identidad de significado entre enunciados normativos y proposiciones normativas, o ii) la equivalencia material entre enunciados normativos y proposiciones normativas. De hecho, es tan débil que es perfectamente compatible con el anti-reduccionismo semántico de HART, KELSEN y RAZ. 
cierto sistema normativo. Esa ha sido su posición clásica. He intentado mostrar que esa posición clásica tiene que ser revisada y que, de hecho, tanto AlCHOURRÓN como BULYGIN han adoptado posiciones más débiles. Con esos progresivos debilitamientos, sin embargo, disminuyen las razones para admitir que la lógica de proposiciones normativas es una formalización adecuada de la lógica subyacente al conocimiento del derecho. 